\title{
Effects of RPE-conditioned medium on the differentiation of hADSCs into RPE cells, and their proliferation and migration
}

\author{
YI ZHANG ${ }^{*}$, DANDAN ZHANG ${ }^{*}$, WEI WEI* ${ }^{*}$ BINGQIAO SHEN, YUYAO WANG, \\ YINGJIE ZHANG, YIDAN ZHANG, JING JI, HAO SUN, MIN LUO and PING GU \\ Department of Ophthalmology, Shanghai Ninth People's Hospital, Shanghai Jiao \\ Tong University School of Medicine, Shanghai 200011, P.R. China
}

Received October 14, 2016; Accepted June 16, 2017

DOI: $10.3892 /$ etm.2017.4997

\begin{abstract}
Age-related macular degeneration (AMD) is associated with the dysfunction and death of the retinal pigment epithelium (RPE). Recently, there has been increasing interest in stem cell-derived RPE cells for cell replacement therapies, such as those for AMD. The present study investigated whether RPE-conditioned medium (RPECM) could promote the differentiation of human adipose tissue-derived mesenchymal stromal cells (hADSCs) into RPE cells, and enhance the proliferation and migration of these cells. Reverse-transcription quantitative polymerase chain reaction analysis demonstrated that RPECM induced hADSCs to differentiate into cells expressing RPE markers, including retinoid isomerohydrolase (RPE65), cytokeratin (CK8) and Bestrophin, which were identified to be significantly upregulated by $\sim 10$-fold, 3.5-fold and 2.4-fold, respectively, compared with the control group [hADSCs cultured in ADSC-conditioned medium (ADSCCM)]. The immunocytochemistry and western blot analysis results demonstrated that the protein levels of RPE65, CK8 and Bestrophin were significantly increased in RPECM-treated hADSCs. In addition, Cell Counting Kit-8 analysis demonstrated that RPECM promoted the proliferation of induced cells. RPECM also increased the expression level of the cell proliferative marker Ki-67. Furthermore, to evaluate the migration potential, cell migration assays were performed. These assays demonstrated that following RPECM treatment hADSCs migrated more quickly compared with the control group. The results of the
\end{abstract}

Correspondence to: Professor Min Luo or Professor Ping Gu, Department of Ophthalmology, Shanghai Ninth People's Hospital, Shanghai Jiao Tong University School of Medicine, 639 Zhizaoju Road, Shanghai 200011, P.R. China

E-mail: qiangson@sh163.net

E-mail: guping2009@126.com

*Contributed equally

Key words: mesenchymal stromal cells, differentiation, proliferation, migration present study suggest that RPECM induces hADSCs to differentiate into RPE cells with higher proliferative and migratory potentials, which may aid in applications for hADSCs in RPE regenerative therapy.

\section{Introduction}

The retinal pigment epithelium (RPE) is an epithelial cell monolayer located between the neural retina and the choroids. The RPE contributes to the long-term preservation of retinal integrity and visual functions by absorbing stray light, transporting nutrients and phagocytosing the shed photoreceptor outer segments (1). Dysfunction of the RPE can result in the loss of functional photoreceptor cells, leading to deterioration or total loss of vision, which serves an important role in the pathogenesis of retinal diseases, including age-related macular degeneration (AMD) (2). AMD is the leading cause of blindness in individuals $>60$ years old, affecting 1.75 million individuals in Western countries (3). There are two types of AMD: Nonexudative AMD and neovascular AMD (4). Nonexudative AMD comprises $85-90 \%$ of all AMD cases, for which there are currently no effective conventional treatments.

A previous study explored the transplantation of RPE cells as a treatment for nonexudative AMD (5). In another study, autologous RPE cells were harvested from the mid-periphery of the retina and transplanted as a cell suspension or patch of RPE into patients with AMD (6). As harvesting autologous RPE cells involves complex surgery with possible sight-threatening complications it is important to consider other donor sources, such as stem cells, for regenerative therapeutics. Recently, a number of studies (7-10) have demonstrated the potential of stem cells to replace damaged retinal cells and improve visual function. The Food and Drug Administration approved the first clinical trial using human embryonic stem cell (hESC)-derived RPE cells for the treatment of nonexudative AMD and Stargardt disease (11). However, ethical issues associated with obtaining hESCs and serious complications, including xenotransplant rejection, have limited the clinical application of this technique $(12,13)$.

Adult induced pluripotent stem cells (iPSCs) possess unlimited self-renewal capacity and can be obtained from the patients' themselves to avoid the risk of rejection and ethical 
issues $(14,15)$. However, certain epigenetic and genetic defects have been detected in iPSCs (16). Therefore, further investigation is required to identify the optimal stem cell type(s) for RPE cell replacement therapy. Bone mesenchymal stem cells (BMSCs) possess multi-differentiation potential (17). BMSCs can undergo osteogenesis, adipogenesis and chondrogenesis differentiation, and can be induced to differentiate into retinal cells and cells from photoreceptor lineages $(18,19)$. However, the specific induction of RPE cells from BMSCs remains in its infancy (20). Additionally, the source of hBMSCs is inadequate and accessing the cells increases the level of pain experienced by patients (21). By contrast, adipose tissue-derived mesenchymal stem cells (ADSCs) possess the following notable advantages: Abundant source and multilineage differentiation capacity, including osteogenesis, chondrogenesis and neurogenesis (22-24). Thus, ADSCs are attractive candidates for cell replacement therapies. A previous study reported that ADSCs could differentiate into neuron-like cells with neuronal markers (24). However, whether hADSCs can be induced to differentiate into RPE cells remains unknown.

The present study investigated the influence of RPE-conditioned medium (RPECM) on the differentiation of hADSCs into RPE cells. The results of the present study revealed that hADSCs incubated with RPECM could differentiate into RPE-like cells, and the proliferation and migration abilities of these induced cells were increased. These results suggest that RPECM-induced hADSCs have potential future applications in retinal degeneration treatment.

\section{Materials and methods}

hADSCs isolation, cultivation and tridifferentiation. The Medical Ethics Committee of the Ninth People's Hospital of Shanghai Jiao Tong University School of Medicine (Shanghai, China) approved the protocols used in the present study. Written informed consent was received from the 4 patients included in the current study. hADSCs were acquired from human subcutaneous adipose tissue: The process of isolation and characterization of mesenchymal stem cells (MSCs) from the subcutaneous adipose tissue acquired from 4 outpatients (healthy adults; aged 20-28 years; 1 male, 3 female) who had undergone blepharoplasties was performed as previously reported (25). Patients were recruited between March and September 2015. Briefly, adipose tissue was digested with $0.2 \%$ collagenase type I (Sigma-Aldrich; Merck KGaA, Darmstadt, Germany) for $2 \mathrm{~h}$ at $37^{\circ} \mathrm{C}$. To obtain stem cells containing high-density stromal vascular fraction (SVF) pellets, the digested adipose tissue was centrifuged at $4^{\circ} \mathrm{C}$ at $1,200 \mathrm{x}$ for $10 \mathrm{~min}$. The resuspended SVF pellets were then plated into culture flasks for $72 \mathrm{~h}$ in MSC growth-promoting medium [Dulbecco's modified Eagle medium/Ham's F-12 Nutrient mixture (DMEM/F12); Gibco; Thermo Fisher Scientific, Inc., Waltham, MA, USA] supplemented with $100 \mathrm{U} / \mathrm{ml}$ penicillin (Beijing Solarbio Science and Technology Co., Ltd., Beijing, China) and 10\% fetal bovine serum (FBS; Gibco; Thermo Fisher Scientific, Inc.) and incubated in a humidified atmosphere at $37^{\circ} \mathrm{C}$ with $5 \% \mathrm{CO}_{2}$. Non-adherent cells were removed by washing the plate $72 \mathrm{~h}$ after plating. The cells that remained were plated in $75 \mathrm{~cm}^{2}$ culture flasks and incubated in a humidified atmosphere at $37^{\circ} \mathrm{C}$ with $5 \% \mathrm{CO}_{2}$. The medium was renewed every 2 days. Once the adherent cells reached 70-90\% confluence, they were removed with $0.25 \%$ trypsin $/ 0.02 \%$ EDTA (Gibco; Thermo Fisher Scientific, Inc.) and passaged at a dilution of 1:2. All the cells used in the experiments were obtained at passage two to three. The morphology of hADSCs was observed by a phase-contrast microscope (Olympus Corporation, Tokyo, Japan). The multipotency characteristics of hADSCs that enable them to differentiate into osteogenic, adipogenic and chondrogenic lineages were assessed, and the osteo-, adipo- and chondro-inductive medium was purchased from Invitrogen (Thermo Fisher Scientific, Inc., Waltham, MA, USA) was added as previously reported (25).

Flow cytometry. hADSCs were trypsinized, washed, suspended in PBS and subsequently blocked in $2 \%$ bovine serum albumin (Sigma-Aldrich; Merck KGaA) at room temperature for $1 \mathrm{~h}$, prior to incubation with PE mouse anti-human antibodies targeting cluster of differentiation (CD)40, CD73, CD9, CD105 (1:200; all from BD Biosciences, San Jose, CA, USA; cat. no. 555589, 550257, 555596 and 560839, respectively) and rabbit polyclonal anti-CD133 (1:200; Abcam, Cambridge, UK; cat. no. ab19898) for 1.5 h at $4^{\circ} \mathrm{C}$. A nonspecific mouse immunoglobulin G (IgG; BD Biosciences; catalogue number: 555749) was substituted for the primary antibodies as an isotype control. hADSCs incubated with rabbit polyclonal anti-CD133 were subsequently stained with a fluorescein isothiocyanate-labeled secondary antibody (1:800; BD Biosciences; cat. no. 553881) for $30 \mathrm{~min}$ at room temperature. The samples were analysed utilizing a FACScan ${ }^{\mathrm{TM}}$ flow cytometer (BD Biosciences), which evaluated 100,000 cells/sample (FlowJo version 7.6; FlowJo LLC, Ashland, OR, USA) (26).

Preparation of RPECM and ADSC-conditioned medium (ADSCCM). ARPE19 RPE cells (American Type Culture Collection, Manassas, VA, USA) were plated into 6-well plates at a density of $1 \times 10^{5}$ cells/well in MSC growth-promoting medium supplemented with $100 \mathrm{U} / \mathrm{ml}$ penicillin and $10 \%$ fetal bovine serum (FBS; Gibco; Thermo Fisher Scientific, Inc.) and incubated in a humidified atmosphere at $37^{\circ} \mathrm{C}$ with $5 \%$ $\mathrm{CO}_{2}$. ARPE19 cells were collected every 2 days as conditioned medium until the cells reached $80-90 \%$ confluence. RPECM was harvested, centrifuged at $4^{\circ} \mathrm{C}, 12,000 \mathrm{x} \mathrm{g}$ for $15 \mathrm{~min}$ to remove solid particles and stored at $-20^{\circ} \mathrm{C}$. The aforementioned method was also used to collect ADSCCM from hADSCs.

RPE differentiation of hADSCs. hADSCs and ARPE19 cells were separately seeded into 6-well plates at a density of $\sim 2 \times 10^{5}$ cells/well and incubated in a humidified atmosphere at $37^{\circ} \mathrm{C}$ with $5 \% \mathrm{CO}_{2}$ overnight in complete DMEM/F-12 medium (the control medium). The present study included the following groups: The hADSC experimental group [ADSCs+RPECM (RPECM was diluted 1:2 with the control medium)], the hADSC negative control group [ADSC+ADSCCM (ADSCCM was diluted 1:2 with the control medium)] and the RPE cell positive control group [RPE+RPECM (RPECM was diluted 1:2 with the control medium)]. For all groups, the culture medium was replaced every 2 days. The cells were grown at $37^{\circ} \mathrm{C}$ with $5 \%$ $\mathrm{CO}_{2}$ for 10 days for the RPE differentiation experiment. At least three independent experiments were performed in duplicate. 
RNA isolation and quality controls. The extraction of total RNA from the cells was performed using TRIzol ${ }^{\mathrm{TM}}$ Reagent (Invitrogen; Thermo Fisher Scientific, Inc.), according to the manufacturer's protocol. DNase I was used to digest and eliminate any contaminating genomic DNA. The concentration and purity of the extracted total RNA were assessed spectrophotometrically at optical densities (ODs) of 260 and $280 \mathrm{~nm}$. The samples with OD 260/280 $\mathrm{nm}$ ratios between 1.9 and 2.1 were used for complementary (c)DNA synthesis.

Reverse transcription-quantitative polymerase chain reaction $(R T-q P C R)$ analysis. Samples of the extracted RNA $(1 \mu \mathrm{g})$ from the cells of each group were reverse transcribed using the PrimeScript ${ }^{\mathrm{TM}}$ RT reagent kit (Perfect Real Time; Takara Biotechnology Co., Ltd., Dalian, China) according to the manufacturer's protocol. Following reverse transcription, the resulting cDNA was diluted 10 -fold in nuclease-free water (Invitrogen; Thermo Fisher Scientific, Inc.) and utilized as a template for qPCRs, which were performed on an Applied Biosystems ${ }^{\circledR} 7500$ Real-Time PCR system (Thermo Fisher Scientific, Inc.). The solutions used in the qPCRs had $(10 \mu \mathrm{l})$ contained $5 \mu \mathrm{l}$ of $2 \mathrm{X}$ Power SYBR ${ }^{\mathrm{TM}}$ Green PCR Master mix (Applied Biosystems; Thermo Fisher Scientific, Inc.), $1 \mu \mathrm{l}$ of diluted cDNA $(1,000 \mathrm{ng} / \mathrm{ul})$ and $300 \mathrm{nmol}$ of gene-specific primers. The primer sequences are presented in Table I. qPCR was performed at $95^{\circ} \mathrm{C}$ for $10 \mathrm{~min}$, followed by 40 cycles of amplification $\left(15 \mathrm{sec}\right.$ at $95^{\circ} \mathrm{C}$ and $1 \mathrm{~min}$ at $60^{\circ} \mathrm{C}$ ). The relative mRNA expression was analysed using the Pfaffl method (27). The relative mRNA levels are expressed as the fold change relative to the negative control following normalization to GAPDH expression.

Western blot analysis. Cells were lysed with radioimmunoprecipitation assay lysis buffer (Beyotime Institute of Biotechnology, Haimen, China) supplemented with $1 \mathrm{mM}$ phenylmethylsulfonyl fluoride (Invitrogen; Thermo Fisher Scientific, Inc.). A bicinchoninic acid assay (Pierce ${ }^{\mathrm{TM}}$ BCA Protein assay kit; Thermo Fisher Scientific,Inc.) and SDS-PAGE were utilized to measure the protein concentrations. Proteins (24 $\mu \mathrm{l} /$ lane) were separated by $10 \%$ SDS-PAGE. Following SDS-PAGE, the proteins were transferred onto polyvinylidene fluoride membranes (EMD Millipore, Billerica, MA, USA). The membranes were blocked with $5 \%$ skimmed milk at room temperature for $1 \mathrm{~h}$, and they were then incubated with rabbit monoclonal antibodies directed against fatty acid binding protein 4 (FABP4), mouse monoclonal antibodies directed against binding sialoprotein (BSP), rabbit polyclonal antibody antibodies directed against sex determining region Y-box 9 (Sox9) (all 1:1,000; Abcam, Cambridge, UK) and mouse monoclonal antibodies directed against retinoid isomerohydrolase (RPE65; 1:200; Santa Cruz Biotechnology, Inc., Dallas, TX, USA) at $4^{\circ} \mathrm{C}$ overnight, and mouse anti- $\beta$-actin antibodies (1:5,000; Sigma-Aldrich; Merck KGaA; cat. no. A1978) at $37^{\circ} \mathrm{C}$ for $2 \mathrm{~h}$. Next, the membranes were incubated with horseradish peroxidase-conjugated secondary antibodies (1:5,000; Sigma-Aldrich; Merck KGaA; cat. no. 12015196001$)$ at $37^{\circ} \mathrm{C}$ for $1 \mathrm{~h}$. Protein bands were visualized using Immobilon western Chemilum HRP substrate (Merck KGaA) and Odyssey software (version 3.0; LI-COR Biosciences, Lincoln, NE, USA). Quantification of the densitometric intensities of the protein bands was performed using Image J software (version 1.47; National Institutes of Health, Bethesda, MD, USA). Data were normalized to $\beta$-actin.

Immunocytochemistry. hADSCs or RPE cells were plated onto $18 \mathrm{~mm}$ glass coverslips at a density of $8 \times 10^{4}$ cells/well (VWR International, Radnor, PA, USA) in 12-well plates. After 2 days in culture, when the cells reached $50-70 \%$ confluence, the cells were fixed with $4 \%$ paraformaldehyde (Sigma-Aldrich; Merck $\mathrm{KGaA}$ ) at room temperature for 15 min, permeabilized with $0.3 \%$ Triton $^{\mathrm{TM}}$ X-100 (Sigma-Aldrich; Merck KGaA) in PBS and blocked with $10 \%$ normal goat serum (NGS; Invitrogen; Thermo Fisher Scientific, Inc.) at room temperature for $1 \mathrm{~h}$. Next, the cells were incubated with mouse monoclonal anti-proliferation marker protein Ki-67 (1:200; BD Biosciences; cat. no. 556003), mouse monoclonal anti-RPE65, mouse monoclonal anti-cytokeratin 8 (CK8) (both 1:50; Santa Cruz Biotechnology, Inc.; cat. nos. sc-73616 and sc-58736) and mouse monoclonal anti-Bestrophin (1:50; Novus Biologicals, LLC, Littleton, CO, USA; cat. no. NB300-164) antibodies at $4^{\circ} \mathrm{C}$ overnight. Thereafter, the cells were incubated with fluorescently-labelled secondary antibodies (1:800; Alexa Fluor ${ }^{\circledR}$ 546 goat anti-mouse; Invitrogen; Thermo Fisher Scientific, Inc.; cat. no. A11030) at room temperature for $1 \mathrm{~h}$, as previously described (28). The cells were then rinsed three times in PBS and the cell nuclei were counterstained with Hoechst 33342 (2 mg/ml; ApexBio Technology, Houston, TX, USA) at room temperature for $5 \mathrm{~min}$.

Cells were cultured for $12 \mathrm{~h}$ in the presence of $10 \mathrm{mM}$ 5-bromo-2-deoxyuridine (BrdU; Sigma-Aldrich; Merck $\mathrm{KGaA}$ ) and fixed in $4 \%$ paraformaldehyde for $15 \mathrm{~min}$ at room temperature. The cells were then washed in PBS, incubated with $2 \mathrm{M} \mathrm{HCl}$ for $30 \mathrm{~min}$ at room temperature and washed with Hanks' Balanced Salt Solution followed by PBS at room temperature. The cells were incubated with blocking buffer (PBS containing 10\% NGS, $0.3 \%$ Triton X-100 and $100 \mathrm{mg} / \mathrm{ml}$ RNaseA) for 60 min at room temperature. Following incubation with anti-BrdU antibodies (1:1,000; Santa Cruz Biotechnology; cat. no. sc-32323) at $4^{\circ} \mathrm{C}$ overnight in the blocking buffer, the cells were washed in PBS and incubated with Alexa Fluor ${ }^{\circledR}$ 546-conjugated goat anti-mouse secondary antibody (1:800; Invitrogen; Thermo Fisher Scientific, Inc.; cat. no. A11030) for $1 \mathrm{~h}$ at room temperature. The cell nuclei were counterstained with $2 \mathrm{mg} / \mathrm{ml}$ Hoechst 33342 at room temperature for $5 \mathrm{~min}$.

The cells were visualized and imaged using a fluorescence microscope (Olympus BX51; Olympus Corporation, Tokyo, Japan). Following the merging of images of immunopositive cells with nuclei stained with Hoechst 33342 and cells treated with antibodies, the percentage of positively-stained cells was counted using Image-Pro Plus software (version 6.0; Media Cybernetics, Inc., Rockville, MD, USA).

Cell Counting Kit (CCK)-8 assay. The effects of RPECM, ADSCCM and complete culture medium (DMEM/F12, $100 \mathrm{U} / \mathrm{ml}$ penicillin and $10 \%$ fetal bovine serum) on the proliferation of hADSCs were assessed using the CCK-8 assay (Dojindo Molecular Technologies, Inc., Kumamoto, Japan). Briefly, the cells were seeded at a density of $1 \times 10^{4}$ cells/well into 96-well plates. On days 0,1 and 2 of culturing in a humidified atmosphere at $37^{\circ} \mathrm{C}$ with $5 \% \mathrm{CO}_{2}, 10 \mu \mathrm{l}$ of CCK-8 solution 
Table I. Primers used for reverse transcription quantitative polymerase chain reactions.

\begin{tabular}{|c|c|c|c|c|c|}
\hline \multirow[b]{2}{*}{ Gene } & \multirow[b]{2}{*}{ Accession no. } & \multicolumn{2}{|c|}{ Primer sequence $\left(5^{\prime}-3^{\prime}\right)$} & \multirow{2}{*}{$\begin{array}{c}\text { Annealing } \\
\text { temperature }\left({ }^{\circ} \mathrm{C}\right)\end{array}$} & \multirow{2}{*}{$\begin{array}{l}\text { Product } \\
\text { size (bp) }\end{array}$} \\
\hline & & Forward & Reverse & & \\
\hline RPE65 & NM_000329 & $\begin{array}{l}\text { GCCAATTTACGTGA } \\
\text { GAACTGGG }\end{array}$ & $\begin{array}{l}\text { CCAGATAGTCTCGT } \\
\text { CACTGCAC }\end{array}$ & 60 & 177 \\
\hline Bestrophin & NM_001139443 & $\begin{array}{l}\text { TCCCACCTGCCTAG } \\
\text { TCGCCA }\end{array}$ & $\begin{array}{l}\text { TTGTAGATGCTGCC } \\
\text { CCGCCA }\end{array}$ & 60 & 186 \\
\hline CK8 & NM_002283 & $\begin{array}{l}\text { TGTGCCTACCTGCG } \\
\text { GAAATC }\end{array}$ & $\begin{array}{l}\text { CTATGACCGAGGTG } \\
\text { TCTGAGA }\end{array}$ & 60 & 133 \\
\hline GAPDH & NM_014364 & $\begin{array}{l}\text { TGTGGGCATCAATG } \\
\text { GATTTGG }\end{array}$ & $\begin{array}{l}\text { ACACCATGTATTCC } \\
\text { GGGTCAAT }\end{array}$ & 60 & 116 \\
\hline ALP & NM_080621 & $\begin{array}{l}\text { GGCCTGTACCATAC } \\
\text { AAGCCC }\end{array}$ & $\begin{array}{l}\text { CCACGTAGACGAG } \\
\text { GTAGTTGTG }\end{array}$ & 60 & 121 \\
\hline BSP & NM_012587.2 & $\begin{array}{l}\text { TGGAGATGCAGAG } \\
\text { GGCAAGGCT }\end{array}$ & $\begin{array}{l}\text { AGTTGGTGCTGGT } \\
\text { GCCGTTGA }\end{array}$ & 60 & 133 \\
\hline FABP4 & NM_001442 & $\begin{array}{l}\text { ACTGGGCCAGGAA } \\
\text { TTTGACG }\end{array}$ & $\begin{array}{l}\text { CTCGTGGAAGTGA } \\
\text { CGCCTT }\end{array}$ & 60 & 183 \\
\hline AdpoQ & NM_198504 & $\begin{array}{l}\text { GGTGCTGAAGCCT } \\
\text { ACCAAC }\end{array}$ & $\begin{array}{l}\text { AGGAAGAACAGAC } \\
\text { GGCAGAAC }\end{array}$ & 60 & 93 \\
\hline Col2A1 & NM_001844 & $\begin{array}{l}\text { TGGACGCCATGAA } \\
\text { GGTTTTC }\end{array}$ & $\begin{array}{l}\text { TGGGAGCCAGATT } \\
\text { GTCATCTC }\end{array}$ & 60 & 183 \\
\hline SOX9 & NM_000346 & $\begin{array}{l}\text { AGCGAACGCACAT } \\
\text { CAAGAC }\end{array}$ & $\begin{array}{l}\text { CTGTAGGCGATCT } \\
\text { GTTGG }\end{array}$ & 60 & 85 \\
\hline
\end{tabular}

RPE65, retinoid isomerohydrolase; CK8, cytokeratin 8.

was added to each well. Following incubation for $4 \mathrm{~h}$ at $37^{\circ} \mathrm{C}$, according to the manufacturer's protocol, the absorbance at a wavelength of $450 \mathrm{~nm}$ was measured using a microplate reader (ELx800 ${ }^{\mathrm{TM}}$; BioTek Instruments, Inc., Winooski, VT, USA).

\section{Cell migration assays}

Wound healing assay. The cells were seeded into 6-well plates at a density of $3 \times 10^{5}$ cells/well and grown into monolayers. Upon reaching 95\% confluence, the cell monolayer was scraped using a pipette tip to generate scratch wounds. To remove floating debris, the cells were washed with PBS. The cells were incubated with ADSCCM or RPECM for 0, 24 or $48 \mathrm{~h}$ in a humidified atmosphere at $37^{\circ} \mathrm{C}$ with $5 \% \mathrm{CO}_{2}$. Images were obtained by using a phase-contrast microscope and the number of cells that had migrated and the total cell number was measured and analysed using ImageJ software (version 1.47; National Institutes of Health, Bethesda, MD, USA). The cell migration rate (\%) was calculated as follows: (Number of cells that migrated/total number of cells) $\times 100$.

Transwell assay. Cells were suspended at a density of $1 \times 10^{5}$ cells $/ \mathrm{ml}$. Then, $0.2 \mathrm{ml}$ of each suspension was added to the top of a Transwell chamber with a polyethylene terephthalate membrane ( $8 \mathrm{~mm}$ pore size; EMD Millipore). Conditioned medium $(0.4 \mathrm{ml})$ supplemented with control medium $(0.2 \mathrm{ml})$ was added to the lower chamber of each well to act as a chemoattractant. The cells were incubated for $24 \mathrm{~h}$ at $37^{\circ} \mathrm{C}$ and those that did not migrate through the pores were removed by scraping the upper surface of the membrane with a cotton swab. The cells that migrated to the lower surface of the membrane were fixed at room temperature for $10 \mathrm{~min}$ in $100 \%$ methanol and stained with $0.1 \%$ crystal violet at room temperature for $5 \mathrm{~min}$. Images were obtained by using a phase-contrast microscope.

Statistical analysis. All data are from at least three independent experiments and are presented as the mean \pm standard deviation. Statistical evaluation of the data was performed using one-way analysis of variance followed by a post hoc Dunnett's multiple comparisons test. $\mathrm{P}<0.05$ was considered to indicate a statistically significant difference.

\section{Results}

hADSCs possess trilineage differentiation potential. hADSCs were adherent to the plastic culture plates and they exhibited typical MSC characteristics, namely a spindle-shaped, fibroblast-like morphology (Fig. 1A). Flow cytometry analysis revealed that the hADSCs were negative for CD40 and CD133. However, hADSCs were positive for CD73 (99.3\%), CD90 (99.4\%) and CD105 (92.3\%), which represent the characteristic phenotype of MSCs. Mouse IgG 
A
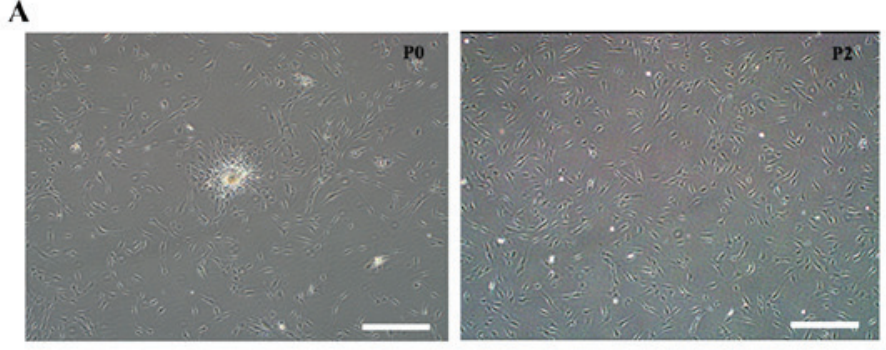

C
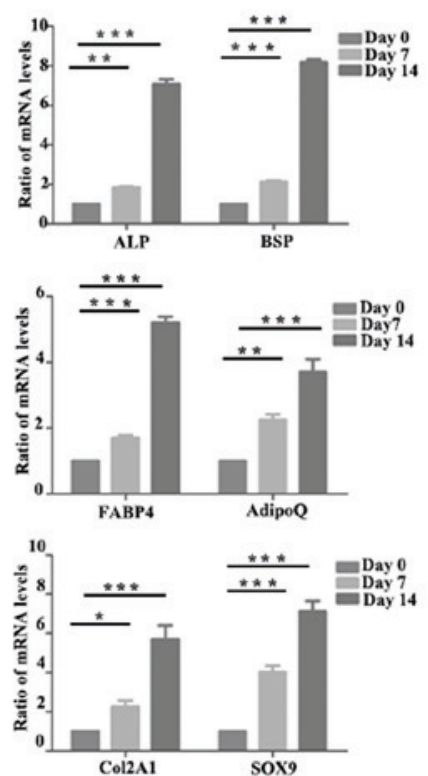

B

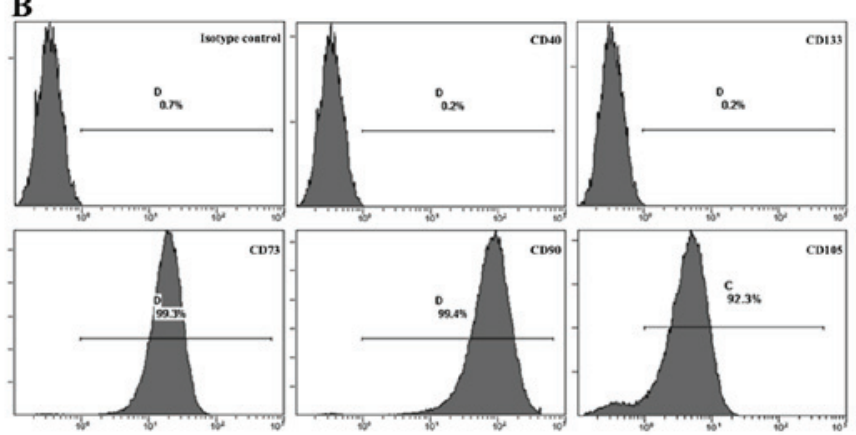

D

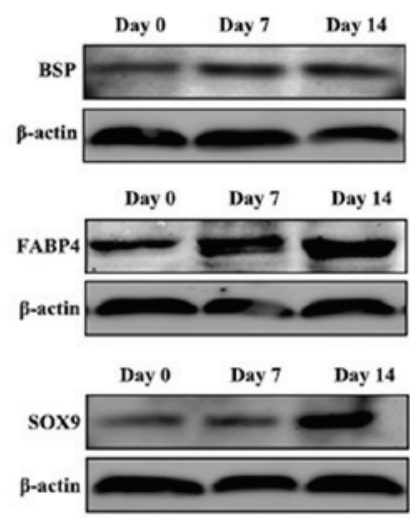

Figure 1. Characteristics of ADSCs. (A) Primary ADSCs exhibited a fibroblast-like morphology as observed under a phase-contrast microscope (Scale bars, $100 \mu \mathrm{m}$.) (B) Flow cytometry analysis demonstrated that ADSCs were positive for CD73, CD90 and CD105, whereas they were negative for CD40 and CD133. (C) Reverse transcription-quantitative polymerase chain reaction analysis demonstrated that the mRNA expression levels of ALP and BSP increased at day 7 and day 14 in ADSCs cultured in osteogenic induction medium, FABP4 and AdipoQ mRNA expression was elevated when cultured in adipogenic induction medium, and mRNA expression levels of Col2A1 and Sox9 were identified to be significantly increased following chondrogenic induction. (D) Western blotting demonstrated that the protein expression levels of BSP, FABP4 and Sox9 were significantly increased during trilineage differentiation. Data from each time point were normalized to Day $0 .{ }^{*} \mathrm{P}<0.05,{ }^{* *} \mathrm{P}<0.01$ and ${ }^{* * * *} \mathrm{P}<0.001$. ADSC, adipose tissue-derived mesenchymal stromal cell; $\mathrm{CD}$, cluster of differentiation; ALP, alkaline phosphatase; BSP, binding sialoprotein; FABP4, fatty acid binding protein 4; AdipoQ, adiponectin, C1Q and collagen domain containing; Col2A1, collagen type II $\alpha$ 1; Sox9, sex determining region Y-box 9.

was used as an isotype control (Fig. 1B). Induction of osteogenesis, adipogenesis and chondrogenesis was performed to analyse the trilineage differentiation potential of these cells. RT-qPCR analysis revealed that the expression levels of osteoblast-specific genes, alkaline phosphatase (ALP) and BSP, were identified to be significantly increased under osteogenic induction (Fig. 1C). Following adipogenic induction, the mRNA expression levels of adipocyte-specific genes, FABP4 and adiponectin (AdipoQ), were also identified to be significantly increased (Fig, 1C). The mRNA expression levels of chondrocyte-specific genes, collagen type II $\alpha 1$ (Col2A1) and Sox9, were identified to be significantly increased when hADSCs were treated with the chondroinductive medium (Fig. 1C). In a parallel approach, western blot analysis demonstrated that the protein expression level of BSP, FABP4 and Sox9 was also enhanced during trilineage differentiation (Fig. 1D). These results demonstrated that hADSCs with trilineage differentiation potentials were successfully harvested.

RPECM promotes the differentiation of hADSCs into RPE cells. There is potential for hADSCs to differentiate into RPE cells, due to their easy isolation, relative abundance, multipotency and rapid expansion. In the present study, the ability of RPECM to enhance the differentiation of hADSCs into RPE cells was evaluated (Fig. 2). RT-qPCR analysis was used to investigate the mRNA expression levels of RPE markers, including RPE65, CK8 and Bestrophin. Compared with the hADSC control group (hADSCs cultured in ADSCCM), RPECM caused a significant $\sim 10$-fold increase in the expression level of RPE65 in hADSCs (Fig. 2A). The mRNA expression levels of CK8 and Bestrophin in hADSCs were also identified to be significantly upregulated in response to RPECM by $\sim 3.5$-fold and 2.4-fold, respectively, compared with the control group (Fig. 2A). Immunocytochemistry was also utilized to evaluate the effects of RPECM on the differentiation of hADSCs into RPE cells. Immunocytochemistry analyses demonstrated that in RPECM-treated hADSCs compared with ADSCCM-treated hADSCs, specific RPE markers were identified to be significantly increased, including RPE65 (69.33 $\pm 2.33 \%)$, CK8 (47.04 $\pm 2.08 \%)$ and Bestrophin $(36.80 \pm 2.08 \%)$ compared with the control group (Fig. 2D and E). In a parallel approach, western blotting demonstrated that the protein expression level of RPE65 was significantly increased compared with the control group when 
A

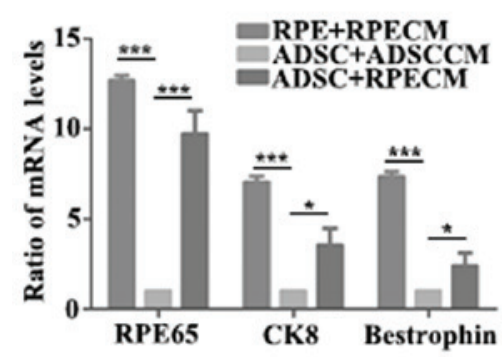

D
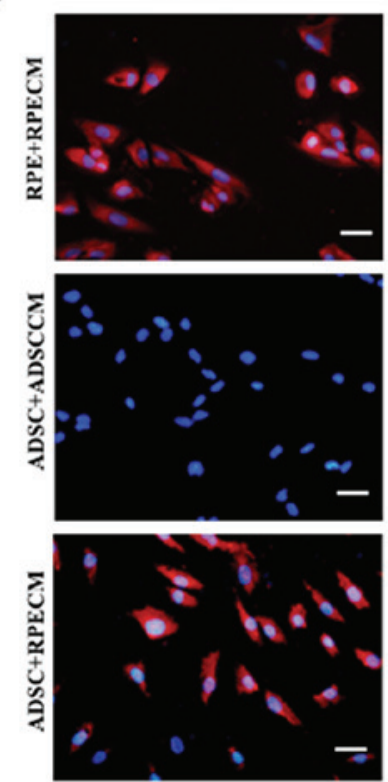

B

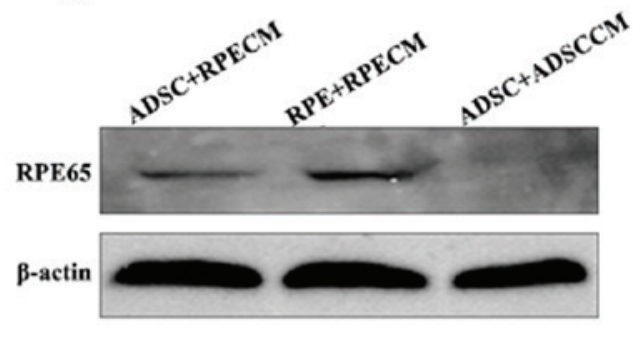

CK8/Hoechst
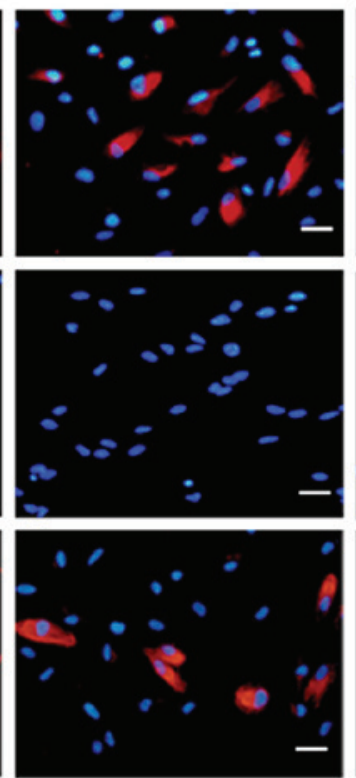

Bestrophin/Hoechst
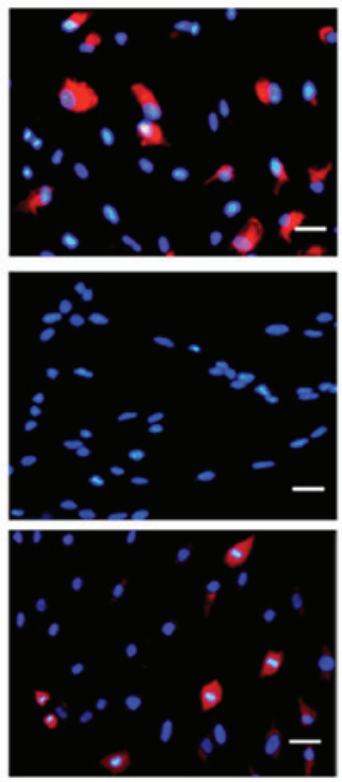

C

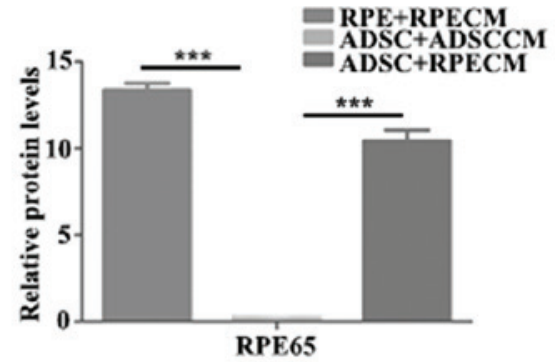

$\mathbf{E}$ RPE+RPECM
ADSC+ADSCCM
ADSC+RPEM

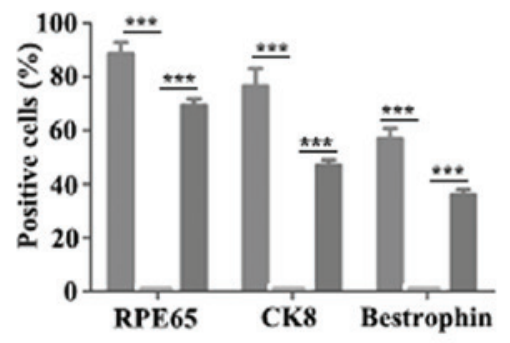

Figure 2. RPECM promotes the differentiation of ADSCs into RPE cells. (A) RT-qPCR results demonstrated that RPECM caused an $\sim 10$-fold increase in the expression level of RPE65 in the treated ADSC group (ADSC+RPECM) in comparison with the ADSC control group (ADSC+ADSCCM). RPE cells were also cultured in RPECM as positive controls (RPE+RPECM). The mRNA expression levels of CK8 and Bestrophin in ADSCs were identified to be upregulated in response to RPECM compared with ADSCCM ( 3.5-fold and $\sim 2$-fold, respectively). (B) Western blotting and (C) western blot analysis demonstrated that the protein expression level of RPE65 also increased when ADSCs were induced with RPECM. (D) Immunocytochemistry and (E) immunocytochemistry analysis was also utilized to evaluate the effects of RPECM on the differentiation of ADSCs into RPE cells. These results were similar to the RT-qPCR and western blotting data; RPE65, CK8 and Bestrophin expression increased in RPECM-treated ADSCs compared to ADSCCM-treated ADSCs (scale bars, $50 \mu \mathrm{m}){ }^{*} \mathrm{P}<0.05,{ }^{* *} \mathrm{P}<0.01,{ }^{* * *} \mathrm{P}<0.001$. RT-qPCR, reverse transcription-quantitative polymerase chain reaction; RPE, retinal pigment epithelium; RPECM, RPE-conditioned medium; ADSC, adipose tissue-derived mesenchymal stromal cell; ADSCCM, ADSC-conditioned medium; CK8, cytokeratin 8; RPE65, retinoid isomerohydrolase.

A

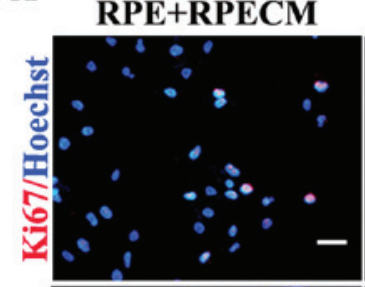

ADSC+ADSCCM
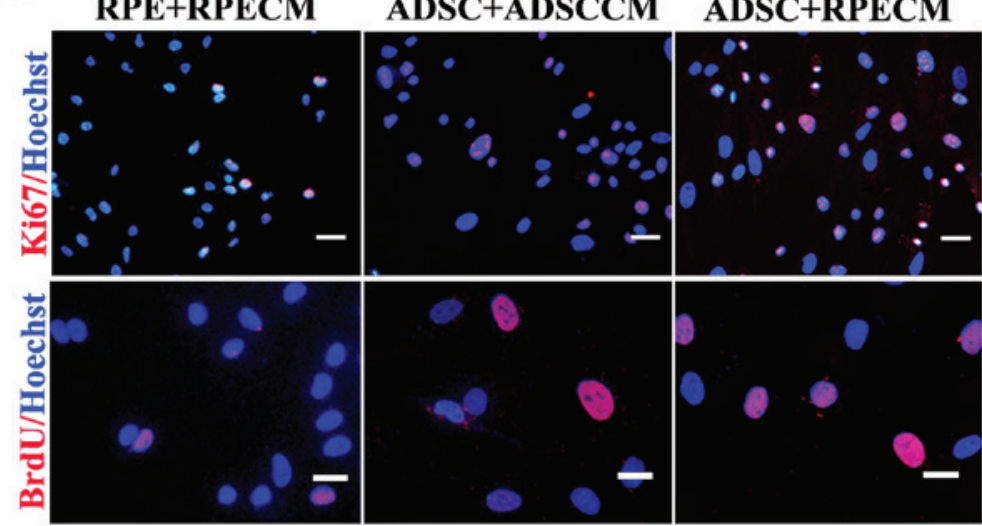

B

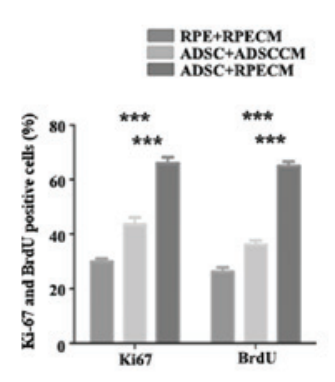

C

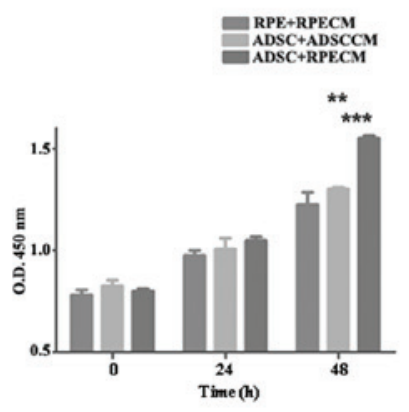

Figure 3. RPECM enhances ADSC proliferation. The proliferation ability of induced ADSCs was assessed by (A) Ki-67/BrdU immunocytochemistry and (B) quantification. The percentage of Ki-67 and BrdU positive cells among the ADSCs incubated with RPECM (ADSC+RPECM) was higher compared with that of ADSC+ADSCCM and RPE+RPECM groups (scale bars, $25 \mu \mathrm{m}$ for BrdU and $50 \mu \mathrm{m}$ for Ki-67). (C) This result was also confirmed by Cell Counting Kit-8 analysis. ${ }^{* *} \mathrm{P}<0.01,{ }^{* * * *} \mathrm{P}<0.001$. RPE, retinal pigment epithelium; RPECM, RPE-conditioned medium; ADSC, adipose tissue-derived mesenchymal stromal cell; ADSCCM, ADSC-conditioned medium; Ki-67, proliferation marker protein Ki-67; BrdU, 5-bromo-2-deoxyuridine; O.D., optical density. 
$\mathbf{A}$

\section{RPE+RPECM}

$\mathbf{0 ~ h}$
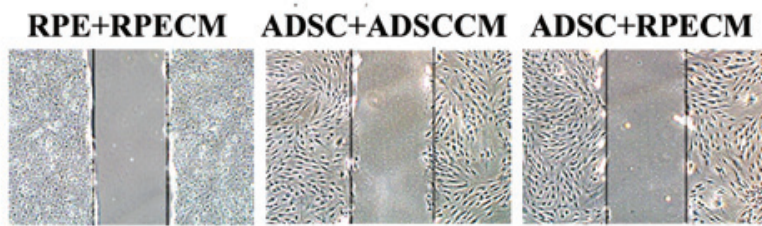

$24 \mathrm{~h}$
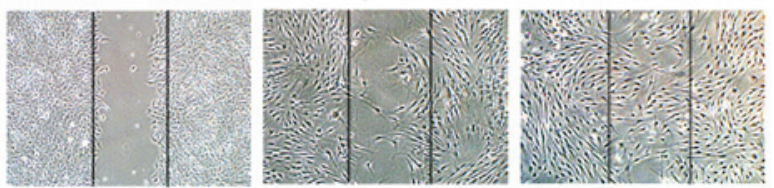

$48 \mathrm{~h}$
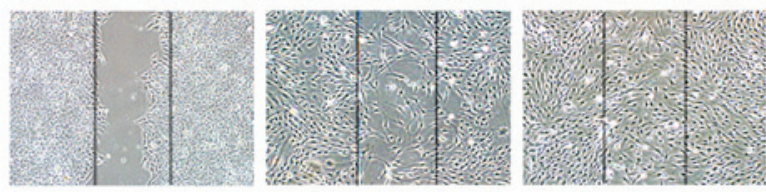

\section{B}

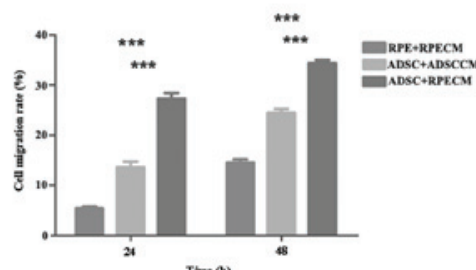

C
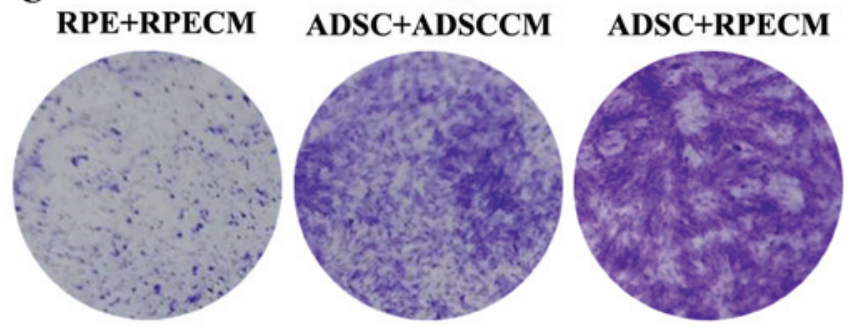

Figure 4. RPECM enhances ADSC migration. (A) A cell migration assay and (B) quantification of the assay was performed on induced cells Compared with ADSCCM treatment, RPECM treatment significantly promoted the migration of ADSCs into the scratched area (scale bars, $100 \mu \mathrm{m}$ ). (C) In a Transwell assay, RPECM-treated ADSCs exhibited a stronger migratory ability compared with the control group. ${ }^{* * *} \mathrm{P}<0.001$ ADSC, adipose tissue-derived mesenchymal stromal cell; RPE, retinal pigment epithelium; RPECM, RPE-conditioned medium; ADSCCM, ADSC-conditioned medium.

hADSCs were induced with RPECM (Fig. 2B and C). These results indicate that RPECM promotes the differentiation of hADSCs into RPE cells.

RPECM enhances hADSC proliferation. The data of the present study demonstrated that RPECM can induce hADSC differentiation into RPE cells. The proliferation potential for these induced cells under RPECM was also explored. Cell proliferation was examined using Ki-67 and BrdU immunocytochemistry, and the CCK-8 assay. The Ki-67 immunocytochemistry results demonstrated that the percentage of Ki-67-positive cells among hADSCs incubated with RPECM was significantly higher compared with that of the hADSC control or the RPE control groups $(65.97 \pm 2.22$, $43.50 \pm 2.57$ and $29.9 \pm 1.86 \%$, respectively; Fig. 3A and B). The immunocytochemistry results for BrdU were similar to that for Ki-67 (Fig. 3A and B). This result was also confirmed by the CCK-8 assay (Fig 3C). The CCK-8 data revealed that there were no marked differences in the proliferation capacity between any two groups at $24 \mathrm{~h}$ of culture. However, after
$48 \mathrm{~h}$, when compared with RPECM-treated RPE cells and ADSCCM-treated hADSCs, the proliferation capacity of RPECM-treated hADSCs was significantly enhanced. These results suggest that RPECM promotes the proliferation of hADSC cells.

RPECM improves hADSC migration. As demonstrated above, hADSCs incubated with RPECM could differentiate into RPE cells and possessed a greater proliferative ability. It is well known that cell migration serves a role in the success or failure of stem cell transplantation. To evaluate the effect of RPECM on cell migration, cell migration assays, including a wound healing assay and Transwell assay were performed. As shown in Fig. 4A and B, compared with ADSCCM treatment, RPECM treatment significantly promoted the migration of hADSCs to the scratched area. The cell migration rate of the hADSCs incubated with RPECM was significantly higher $(34.43 \pm 0.57 \%)$ compared with the hADSC $(24.47 \pm 0.80 \%)$ and RPE cell (14.57 $\pm 0.63 \%$ ) control groups (Fig. 4B). According to the Transwell assay, RPECM-treated hADSCs exhibited a stronger migratory ability compared with those of the control groups (Fig. 4C). These results indicate that following RPECM induction, hADSCs exhibit more potential for migration, which may make them ideal candidates for RPE replacement therapy.

\section{Discussion}

The RPE is physiologically essential for the function of retinal neurons. Degeneration of RPE cells is one cause of the loss of macular function in AMD, a common cause of blindness and major public health issue in the industrialized world. One of the current treatments for AMD is RPE replacement therapy. However, there are limited sources of RPE cells, highlighting the necessity to develop stem cell-derived RPE cells. Recently, ESCs and iPSCs have been proposed as sources of RPE cells for regenerative medicine $(7,29,30)$. However, there are disadvantages; for instance, the use of ESCs raises ethical concerns, while the complications that arise when reprogramming iPSCs limit their clinical application. Therefore, the present study aimed to identify an abundant source of RPE cells with autologous availability, in addition to exploring valid methods for acquiring RPE cells.

In the present study, hADSCs were identified as attractive candidates for cellular therapies since they are autologously available, expandable and exhibit broad differentiation potential, including osteogenesis, chondrogenesis and neurogenesis. As a result, hADSCs were selected as a source of RPE cells, and their potential differentiation into RPE cells was explored. To induce hADSCs to differentiate, part of the microenvironment in which RPE cell differentiation occurs was mimicked by incubating the cells with RPECM. The results of the present study revealed that in RPECM, hADSCs differentiated into RPE-like cells. These induced hADSCs expressed RPE markers, including CK8, Bestrophin and RPE65, and demonstrated an increased proliferative and migratory ability. The RPE differentiation state of the hADSCs was characterized by their morphology and expression of RPE markers in accordance with current scientific knowledge. CK8 is an epithelial cell marker and also an RPE marker (31). Bestrophin, which 
is expressed in the basolateral membrane of RPE, forms calcium-sensitive chloride channels (32). RPE65, which is required for the maintenance of photoreceptor visual cycles and the regeneration of visual pigments by photoreceptors, is also expressed by RPE cells (33). The expression of RPE65, a rate-limiting enzyme for the visual cycle, decreases following two to three passages in RPE cell culture (34). Thus, for use in clinical therapy, hADSCs induced with RPECM present a significant advantage due to their high expression levels of RPE65.

At present, the lack of RPE cell sources restricts cell replacement therapy. The enhancement of proliferation demonstrated in the present study allows the production of abundant RPE cells for transplantation. Suspension transplants have been frequently used in rodent models and ongoing clinical studies $(8,10)$. Cell migration, which allows cells to spread out in the damaged site, is a prerequisite for cell-based replacement therapy. Cell migration is closely associated with successful cell transplantation. The present study demonstrated that following RPECM incubation, hADSCs exhibited increased migration compared with the hADSC and RPE control groups; this may allow an improved success rate of transplantation in vivo.

In conclusion, the present study demonstrated that RPECM could induce the differentiation of hADSCs into RPE-like cells, with enhanced proliferation and migration. These findings indicate that RPECM-induced hADSCs are candidates for efficient RPE replacement therapy. However, whether these RPECM-induced hADSCs can incorporate into the RPE layer and are functional in vivo requires further investigation.

\section{Acknowledgements}

The present study was supported by the National High Technology Research and Development 863 Program (grant no. 2015AA020311), the National Natural Science Foundation of China (grant nos. 81570883, 31300810 and 31500835), the Science and Technology Commission of Shanghai (grant no. 14JC1493103) and the Education Commission of Shanghai (grant no. 14ZZ115).

\section{References}

1. Strauss $O$ : The retinal pigment epithelium in visual function. Physiol Rev 85: 845-881, 2005.

2. Al-Shamekh S and Goldberg JL: Retinal repair with induced pluripotent stem cells. Transl Res 163: 377-386, 2014.

3. Sachdeva MM and Eliott D: Stem cell-based therapy for diseases of the retinal pigment epithelium: From bench to bedside. Semin Ophthalmol 31: 25-29, 2016.

4. Mehta S: Age-related macular degeneration. Prim Care 42: 377-391, 2015.

5. Lee E and MacLaren RE: Sources of retinal pigment epithelium (RPE) for replacement therapy. Br J Ophthalmol 95: 445-449, 2011.

6. Siqueira RC: Autologous transplantation of retinal pigment epithelium in age related macular degeneration. Arq Bras Oftalmol 72: 123-130, 2009 (In Portuguese).

7. Lane A, Philip LR, Ruban L, Fynes K, Smart M, Carr A, Mason C and Coffey P: Engineering efficient retinal pigment epithelium differentiation from human pluripotent stem cells. Stem Cells Transl Med 3: 1295-1304, 2014.
8. Guan Y, Cui L, Qu Z, Lu L, Wang F, Wu Y, Zhang J, Gao F, Tian H, Xu L, et al: Subretinal transplantation of rat MSCs and erythropoietin gene modified rat MSCs for protecting and rescuing degenerative retina in rats. Curr Mol Med 13: 1419-1431, 2013.

9. Lu B, Malcuit C, Wang S, Girman S, Francis P, Lemieux L, Lanza $R$ and Lund R: Long-term safety and function of RPE from human embryonic stem cells in preclinical models of macular degeneration. Stem Cells 27, 2126-2135, 2009.

10. Lund RD, Wang S, Klimanskaya I, Holmes T, Ramos-Kelsey R, Lu B, Girman S, Bischoff N, Sauvé Y and Lanza R: Human embryonic stem cell-derived cells rescue visual function in dystrophic RCS rats. Cloning Stem Cells 8: 189-199, 2006.

11. Mu Y,Zhao M and Su G: Stem cell-based therapies for age-related macular degeneration: Current status and prospects. Int J Clin Exp Med 7: 3843-3852, 2014

12. Cohen CB: Ethical and policy issues surrounding the donation of cryopreserved and fresh embryos for human embryonic stem cell research. Stem Cell Rev 5: 116-122, 2009.

13. Drukker M: Immunogenicity of embryonic stem cells and their progeny. Methods Enzymol 420: 391-409, 2006.

14. Takahashi $\mathrm{K}$ and Yamanaka S: Induction of pluripotent stem cells from mouse embryonic and adult fibroblast cultures by defined factors. Cell 126: 663-676, 2006.

15. Gong J, Fields MA, Moreira EF, Bowrey HE, Gooz M, Ablonczy Z and Del Priore LV: Differentiation of human protein-induced pluripotent stem cells toward a retinal pigment epithelial cell fate. PLoS One 10: e0143272, 2015.

16. Yamanaka S: Induced pluripotent stem cells: Past, present and future. Cell Stem Cell 10: 678-684, 2012.

17. Hsu SH, Kuo WC, Chen YT, Yen CT, Chen YF, Chen KS, Huang WC and Cheng $\mathrm{H}$ : New nerve regeneration strategy combining laminin-coated chitosan conduits and stem cell therapy. Acta Biomater 9: 6606-6615, 2013.

18. Chiou SH, Kao CL, Peng CH, Chen SJ, Tarng YW, Ku HH, Chen YC, Shyr YM, Liu RS, Hsu CJ, et al: A novel in vitro retinal differentiation model by co-culturing adult human bone marrow stem cells with retinal pigmented epithelium cells. Biochem Biophys Res Commun 326: 578-585, 2005.

19. Li N, Li XR and Yuan JQ: Effects of bone-marrow mesenchymal stem cells transplanted into vitreous cavity of rat injured by ischemia/reperfusion. Graefes Arch Clin Exp Ophthalmol 247: 503-514, 2009.

20. Yang LL, Zhou QJ, Wang Y and Wang YQ: Differentiation of human bone marrow-derived mesenchymal stem cells into neural-like cells by co-culture with retinal pigmented epithelial cells. Int J Ophthalmol 3: 23-27, 2010.

21. Jiang Y, Jahagirdar BN, Reinhardt RL, Schwartz RE, Keene CD, Ortiz-Gonzalez XR, Reyes M, Lenvik T, Lund T, Blackstad M, et al: Pluripotency of mesenchymal stem cells derived from adult marrow. Nature 418: 41-49, 2002.

22. Schäffler A and Büchler C: Concise review: Adipose tissue-derived stromal cells-basic and clinical implications for novel cell-based therapies. Stem Cells 25: 818-827, 2007.

23. Kornicka K, Marycz K, Tomaszewski KA, Marędziak M and Śmieszek A: The effect of age on osteogenic and adipogenic differentiation potential of human adipose derived stromal stem cells (hASCs) and the impact of stress factors in the course of the differentiation process. Oxid Med Cell Longev 2015: 309169, 2015.

24. Han C, Song L, Liu Y, Zou W, Jiang C and Liu J: Rat cortex and hippocampus-derived soluble factors for the induction of adipose-derived mesenchymal stem cells into neuron-like cells. Cell Biol Int 38: 768-776, 2014.

25. Kern S, Eichler H, Stoeve J, Klüter H and Bieback K: Comparative analysis of mesenchymal stem cells from bone marrow, umbilical cord, or adipose tissue. Stem Cells 24: 1294-1301, 2006.

26. González-Fernández ML, Pérez-Castrillo $S$, Ordás-Fernández $P$, López-González ME, Colaço B and Villar-Suárez V: Study on viability and chondrogenic differentiation of cryopreserved adipose tissue-derived mesenchymal stromal cells for future use in regenerative medicine. Cryobiology 71: 256-263, 2015.

27. Pfaffl MW: A new mathematical model for relative quantification in real-time RT-PCR. Nucleic Acids Res 29: e45, 2001.

28. Hu Y, Ji J, Xia J, Zhao P, Fan X, Wang Z, Zhou X, Luo M and Gu P: An in vitro comparison study: The effects of fetal bovine serum concentration on retinal progenitor cell multipotentiality. Neurosci Lett 534: 90-95, 2013. 
29. Pennington BO, Clegg DO, Melkoumian ZK and Hikita ST: Defined culture of human embryonic stem cells and xeno-free derivation of retinal pigmented epithelial cells on a novel, synthetic substrate. Stem Cells Transl Med 4: 165-177, 2015.

30. Geng Z, Walsh PJ, Truong V, Hill C, Ebeling M, Kapphahn RJ, Montezuma SR, Yuan C, Roehrich H, Ferrington DA and Dutton JR: Generation of retinal pigmented epithelium from iPSCs derived from the conjunctiva of donors with and without age related macular degeneration. PLoS One 12: e0173575, 2017.

31. Hunt RC and Davis AA: Altered expression of keratin and vimentin in human retinal pigment epithelial cells in vivo and in vitro. J Cell Physiol 145: 187-199, 1990.
32. Milenkovic A, Brandl C, Milenkovic VM, Jendryke T, Sirianant L, Wanitchakool P, Zimmermann S, Reiff CM, Horling F, Schrewe $\mathrm{H}$, et al: Bestrophin 1 is indispensable for volume regulation in human retinal pigment epithelium cells. Proc Natl Acad Sci USA 112: E2630-E2639, 2015.

33. Huang J, Possin DE and Saari JC: Localizations of visual cycle components in retinal pigment epithelium. Mol Vis 15: 223-234, 2009.

34. Alge CS, Suppmann S, Priglinger SG, Neubauer AS, May CA, Hauck S, Welge-Lussen U, Ueffing $M$ and Kampik A: Comparative proteome analysis of native differentiated and cultured dedifferentiated human RPE cells. Invest Ophthalmol Vis Sci 44: 3629-3641, 2003. 